\title{
Toxic Epidermal Necrolysis
}

\section{Induced by Carbamazepine: A Case Report}

\author{
Mochammad Ayyub Arachman ${ }^{\mathrm{a}}$, Sylvia Anggraeni ${ }^{\mathrm{b}}$, Damayanti ${ }^{\mathrm{b}}$, Cita Rosita Sigit \\ Prakoeswa $^{\mathrm{b}}$, Muhammad Yulianto Listiawan ${ }^{\mathrm{b}}$ \\ ayyub.arachman@gmail.com
}

${ }^{a}$ Resident, Dermatology and Venereology Department Faculty of Medicine Universitas Airlangga, Dr. Soetomo Teaching Hospital Jl. Prof. dr. Moestopo 6-8, Surabaya 60286, Indonesia

${ }^{b}$ Dermatologist, Dermatology and Venereology Department Faculty of Medicine Universitas Airlangga, Dr. Soetomo Teaching Hospital Jl. Prof. dr. Moestopo 6-8, Surabaya 60286, Indonesia

\begin{abstract}
Background: Toxic epidermal necrolysis (TEN) is an uncommon but potentially fatal condition. TEN is distinguished by apoptosis of keratinocytes, which results in erosion of the mucous membrane and epidermal detachment.

Case: A man, 20-year-old, came to hospital with skin sore and blisters in almost over his body, accompanied by swollen eyes, sore on the lips and genital. Dermatological examination on the regio trunk showed erythematous and hyperpigmented macules, irregularly shaped, multiple flaccid bullae, and epidermal detachment. The suspected drug, carbamazepine was discontinued immediately, then the patient was given appropriate supportive treatment with intravenous fluid drop (IVFD), systemic corticosteroid, antibiotic, and topical treatment. After 10 days, skin recovery and reepithelialization mucosal was stabilized. The patient was discharged after 12 days if admission. Drug patch testing was performed resulting in positive result for carbamazepine.

Conclusion: The accurate diagnosis, avoidance of the use causative drug and rapid treatment may improve the prognosis. Drug patch testing with positive reactions have high predictive value to avoid using that drug in the future.
\end{abstract}

Keywords: toxic epidermal necrolysis; carbamazepine; drug patch test

\section{Introduction}

Toxic epidermal necrolysis (TEN) is a most commonly caused skin and mucous membrane reaction (Valeyrie \& Roujeau, 2012). TEN is distinguished by apoptosis of keratinocytes leading to erosions of the mucosal membrane and the epidermal separation; separation of less than $10 \%$ of the total surface area of the body is defined by SJS; when it is above $30 \%$ the overlap in TEN is called SJS. TEN is defined as intermediate cases (Mockenhaupt, 2014). It can cause by anticonvulsant drugs, especially the aromatic anticonvulsants such as carbamazepine. Genetic susceptibility may play an important role in the development of TEN to a few "highrisk" medications. A strong link between the human leukocyte antigen HLA-B*1502 and TEN induced by carbamazepine has been observed in Chinese Han from Taiwan. The association between carbamazepineinduced TEN and HLA-B*1502 was not present in European patients who do not have Asian ancestry (Suvichapanich et al., 2015). Most patients with seizure receive anticonvulsants as therapy and carbamazepine being the most commonly used drug that have been enlisted as high-risk drugs for causing TEN (Azfar, 2010). 


\section{Case Presentation}

A man, 20-year-old, came to emergency unit Dr. Soetomo Hospital with chief complaint of skin sore and blisters in almost over his body for 2 days before hospitalized, accompanied by swollen eyes, sore on the lips and genital. The Prodromal complaints appeared (malaise, fever, sore throat). Flaccid blister on his body appeared since 2 days before hospitalization then spread to the extremity, face, and around the outer genitalia The blister containing clear fluid, easy to be broken became more severe, accompanied with pain while swallowing, teary eyes, erosions on the lips-mouth and on genital. Previously, he had history of epilepsy and treated with carbamazepine. After 2 weeks of carbamazepine therapy, erythematous lesions and blisters were observed, detachment of the skin spreading to the entire body. There was a history of applying topical ointment for his rash about two weeks before but no improvement. There was no history of taking any traditional medicine before. There was no history of food or drug allergy. Dermatological examination on the regio trunk showed erythematous and hyperpigmented macules, irregularly shaped, multiple flaccid bullae, and epidermal detachment $>30 \%$ BSA. There was conjunctival lesion hyperemia and secret, erosion on lips hemorrhagic crust and oral mucous membrane, and also erosions on the genital area.

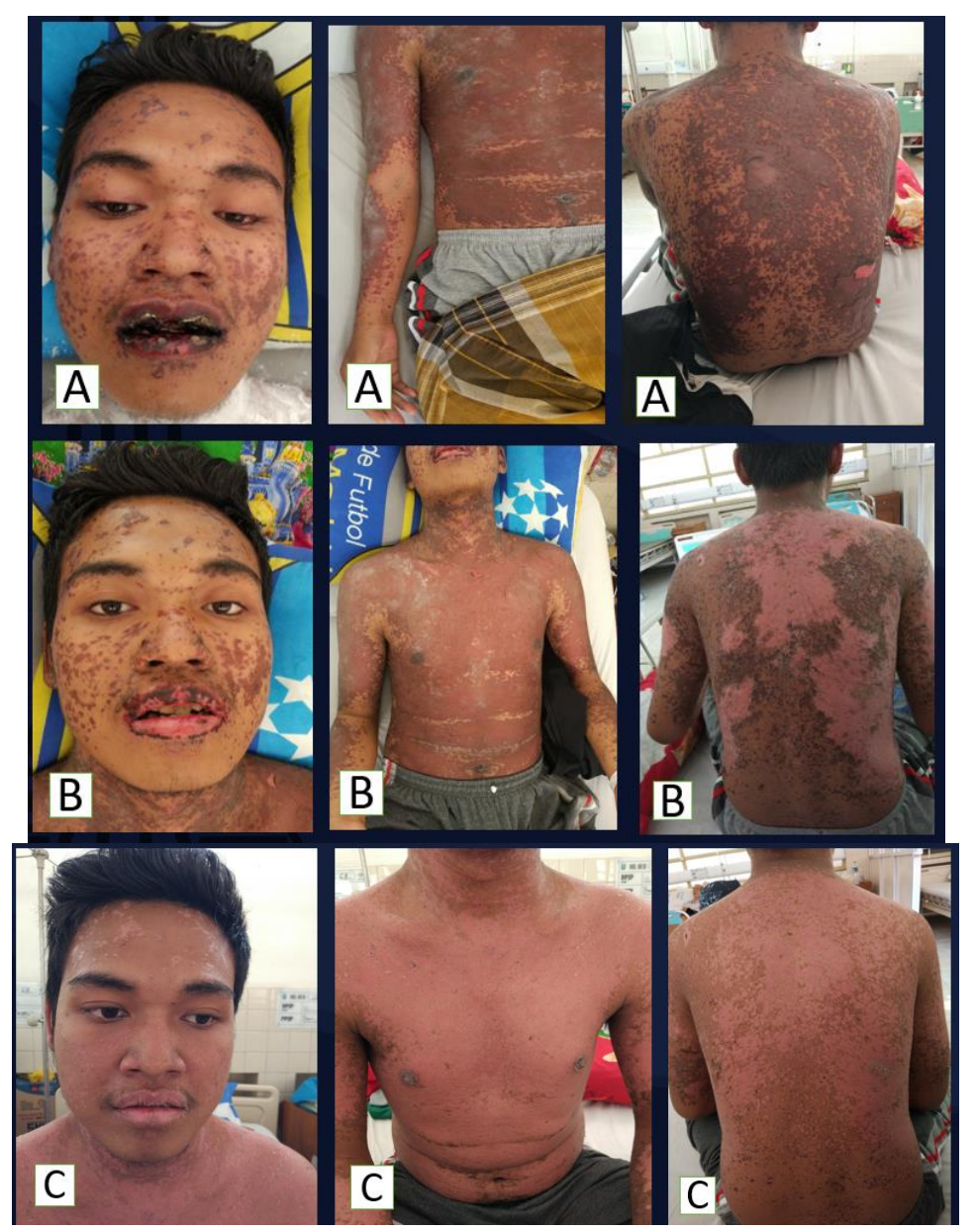

Fig. 1. (a) 3rd day of treatment; (b) 7th day of treatment; (c) 12th day of treatment, lesions are getting better 
The SCORTEN of this patient was 1 . The suspected drug, carbamazepine was discontinued immediately, then the patient was given appropriate supportive treatment with intravenous fluid drop (IVFD), systemic corticosteroid, antibiotic, and topical treatment. After 10 days, skin recovery and reepithelialization mucosal was stabilized. The patient was discharged after 12 days if admission. Drug patch testing was performed resulting in positive result for carbamazepine.

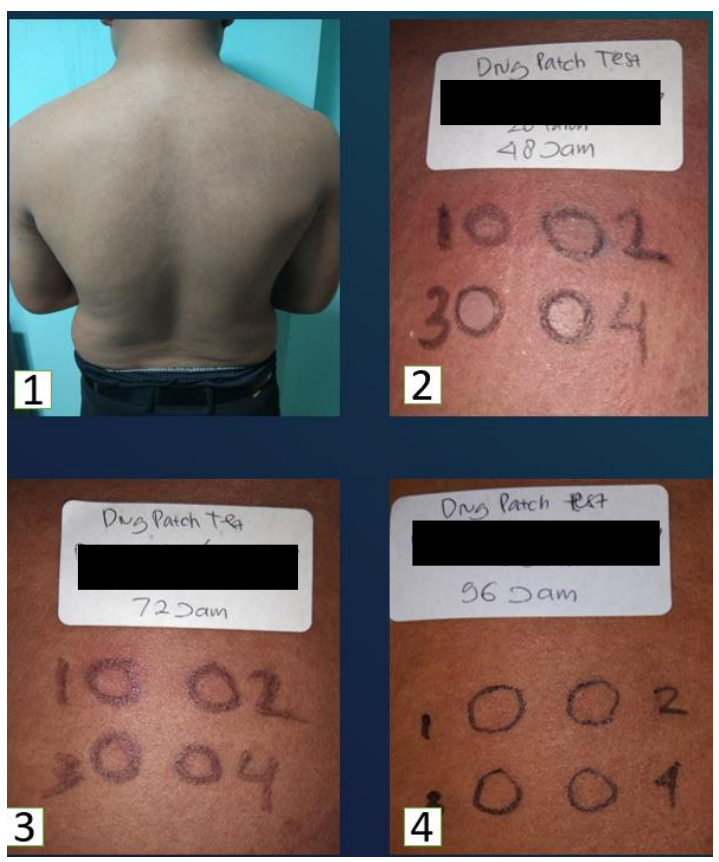

Fig. 2. Result of the drug patch test using carbamazepin. It was performed 6 months after discharged from the hospital. (1) Clinical presentation right before the drug patch test was performed. (2) 48 Hours after patch test. (3) 72 Hours after patch test, +2 result for carbamazepine (4) 96 Hours after patch test

\section{Discussion}

TEN is one of severe complication in patients with epilepsy that receiving anticonvulsants. Based on the history, physical and laboratory examination, the diagnosis of TEN due to carbamazepine was established.

The clinical manifestations were similar to the literature revealed, initially, the eruption was symmetrically spread over the face, upper trunk and next part of extremities. The distal portions of the extremities are relatively spared. But the rash can rapidly extend to the rest of the body within a few days and even a few hours (Valeyrie $\&$ Roujeau, 2012). The first skin damage is marked by irregularly shaped erythematous, dusky red, purpuric macules that gradually coalesce. Nikolsky's sign is positive in erythematous zones was less than $10 \%$. The scope of the epidermal separation lesions is classified into one of the following three groups: (1) SJS, below $10 \%$ of the area of the body (BSA); SJS/TEN, overlap of between 10-30\%; TEN, below $30 \%$ of BSA (Suvichapanich et al., 2015).

The severity and pronouncement of the disease should be determined so as to define the appropriate medical setting for future management once the SJS/TEN diagnosis has been established (Michaels \& Del Roso, 2009). The validated SCORTEN disease severe scoring system can be used to evaluate the prognosis in patients with 
SJS or TEN. Only patients with limited skin involvement, a SCORTEN score of 0 or 1 , and a disease that is not rapidly progressing can be treated in nonspecialized wards. Where possible, a patient with an Intensive Care Unit with the SCORTEN 3 or older should be managed (Valeyrie \& Roujeau, 2012).

The main difference between SJS and TEN appears to be the presence of epidermal detachment (Patel et al., 2013).

Management of SJS/TEN consists of symptomatic treatment, specific treatment in acute stage and also sequele treatment. The mainstay of treatment in SJS/TEN is symptomatic and supportive care which includes fluids and electrolyte replacement, early nutritional support, control of infection, topical skin care and eye care. Whereas specific treatment consists of: corticosteroids, intravenous immunoglobulin, cyclocporine, plasmapheresis or hemodialysis, antitumor necrosis factor agents and also treatment of sequele (Mockenhaupt, 2014). Stopped suspected drug immediately, appropriate supportive treatment, corticosteroid, antibiotic, and topical treatment gave excellent result for this patient. The diagnosis was established by history taking, physical and laboratory examination, and also identify the causative drug which had been confirmed by drug patch test (DPT) (Friedmann \& Arden, 2010). Based on the DPT result, the drugs inducing TEN in this patient were carbamazepine (2+).

\section{Conclusion}

In TEN accurate diagnosis, avoidance of use causative drug and rapid prompt treatment may improve the prognosis. Drug patch testing with positive reactions have high predictive value to avoid using causative drug in the future.

\section{Acknowledgements}

I would like to present my gratitude to all lecturers of Dermatology and venereology at the Faculty of Medicine, Universitas Airlangga, my family, and friends for their continued support and prayers.

\section{References}

Azfar NA, Zia MA, Malik LM, Khan AR, Jahangir M. 2010. Role of Systemic Steroids in The Outcome of Stevens-Johnson Syndrome and Toxic Epidermal Necrolysis. Journal of Pakistan Association of Dermatologist, 20, 158-62.

Friedmann PS, Ardern MJ. 2010. Patch testing in drug allergy. Curr Opin Allergy Clin Immunol, Aug 10(4), $291-6$.

Michaels B, Del Roso JQ. 2009. The Role of Corticosteroid Systemic Therapy in Erythema Multiforme Major and Stevens-Johnson Syndrome (A review of Past and Current Oppinions). J Clin Aesthet Dermatol, 2(31), 51-55.

Mockenhaupt. 2014. Stevens-Johnson syndrome and toxic epidermal necrolysis: clinical patterns, diagnostic considerations, etiology, and therapeutic management. SDER J., 33, 10-15.

Patel K, Barvallaya M, Sharma D, Tripathi C. 2013. A systematic review of the drug-induced Stevens Johnson syndrome and toxic epidermal necrolysis in Indian Population. IJDVL, 79(3), 389-98.

Suvichapanich S, Jittikoon J, Wichukchinda3 N, Kamchaisatian W, Visudtibhan A. 2015. Association analysis of CYP2C9*3 and phenytoin-induced severe cutaneous adverse reactions (SCARs) in Thai epilepsy children. Journal of Human Genetics, 60, $413-417$.

Valeyrie-Allanore L, Roujeau J-C. 2012. Epidermal necrolysis (Stevens-Johnson syndrome and toxic epidermal necrolysis. In: Goldsmith LA, Katz SI, Gilchrest BA, Paller AS, Leffell DJ, Wolff K, editors. Fitzpatrick's dermatology in general medicine. 8th ed. (p.43948) New York: Mc Graw Hill. 